\title{
ASPECT score and Transcranial Doppler flow parameters in middle cerebral artery in acute brain ischaemia.
}

J. Okrój-Lubecka MD¹, J. Balicki Md PhD¹, E. Szurowska MD PhD², G. Kozera MD PhD³.

${ }^{1}$ Hospital in Słupsk, Department of Neurology, Słupsk, Poland.

'Medical University of Gdańsk, II Department of Radiology, Gdańsk, Poland.

${ }^{3}$ Collegium Medicum in Bydgoszcz- Nicolaus Copernicus University in Toruń, Department of Neurology, Bydgoszcz, Poland.

Transcranial Doppler (TCD,TCCD) flow parameters assessed with Thrombolysis in Brain Ischaemia (TIBI) (Fig. 1) score may reflect early brain hypoperfusion. Alberta Stroke Programme early CT Score (ASPECT) $[3,4]$ may also reveal acute brain ischaemia. However, little is known about the relationship between the above parameters. Thus, our goal was to assess the relationship between TCD/TCCD flow parameters in middle cerebral artery (MCA) and ASPECT score in patients with stroke in MCA territory.

Materials and methods: 80 patients with acute MCA ischemia (66 with stroke, mean age $68 \mathrm{yrs}$ and 14 with TIA mean age $68 \mathrm{yrs}$ ) were examined (Tab.1).

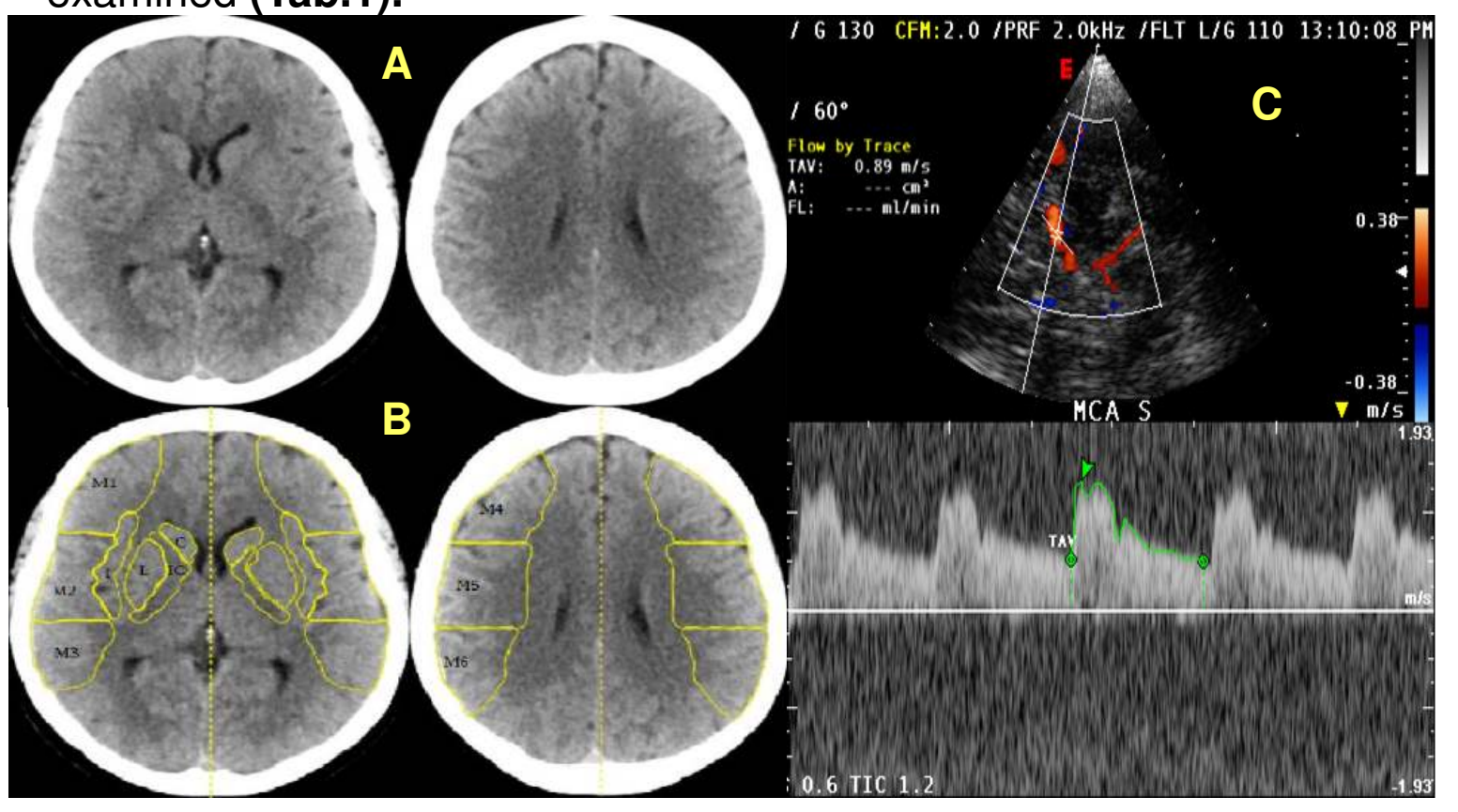

Figure 2. A NCCT B EASPECT 10 pts C TCD TIBI score -5

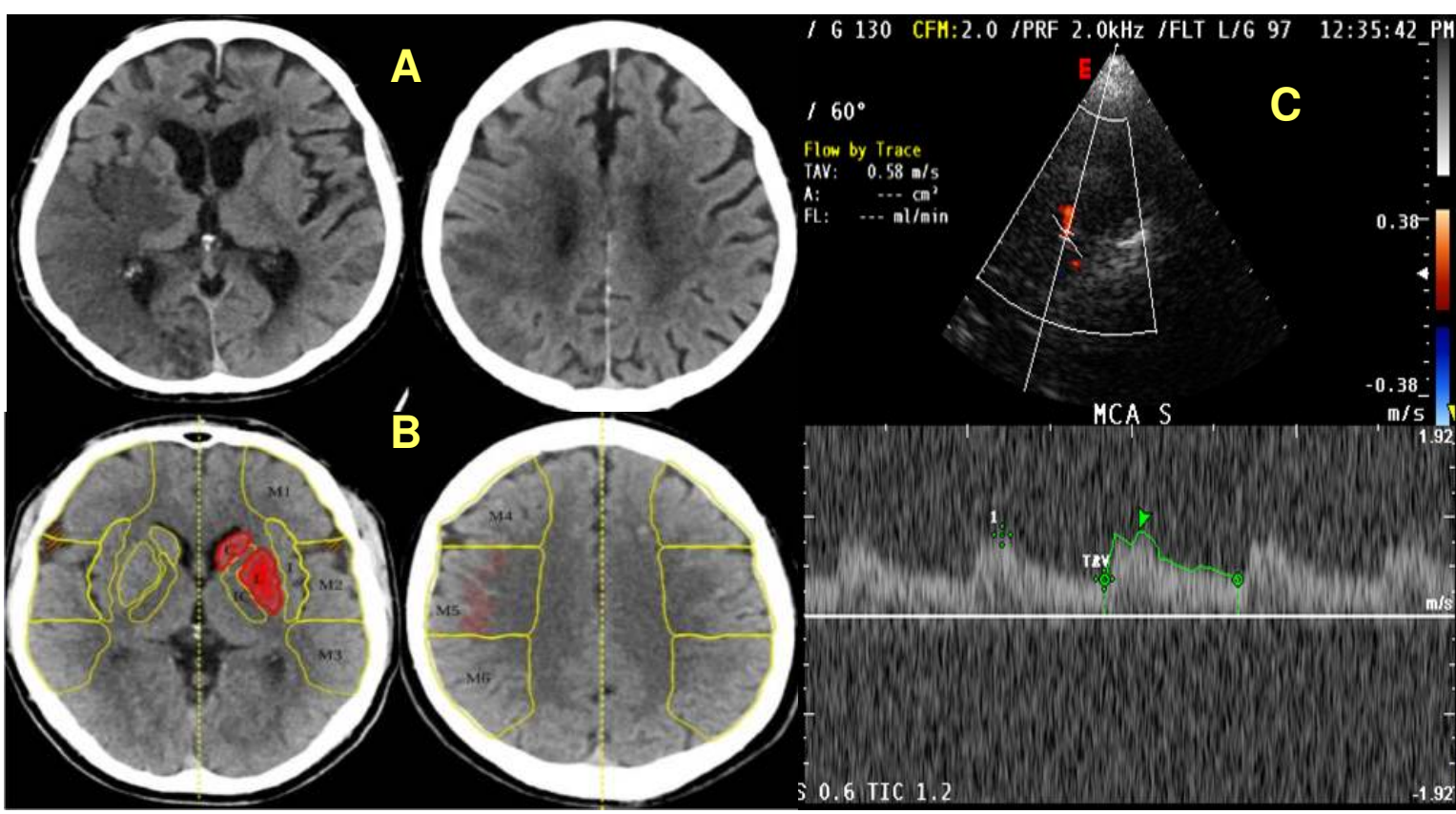

Figure 3. A NCCT B EASPECT 8 pts C TCD TIBI score - 3

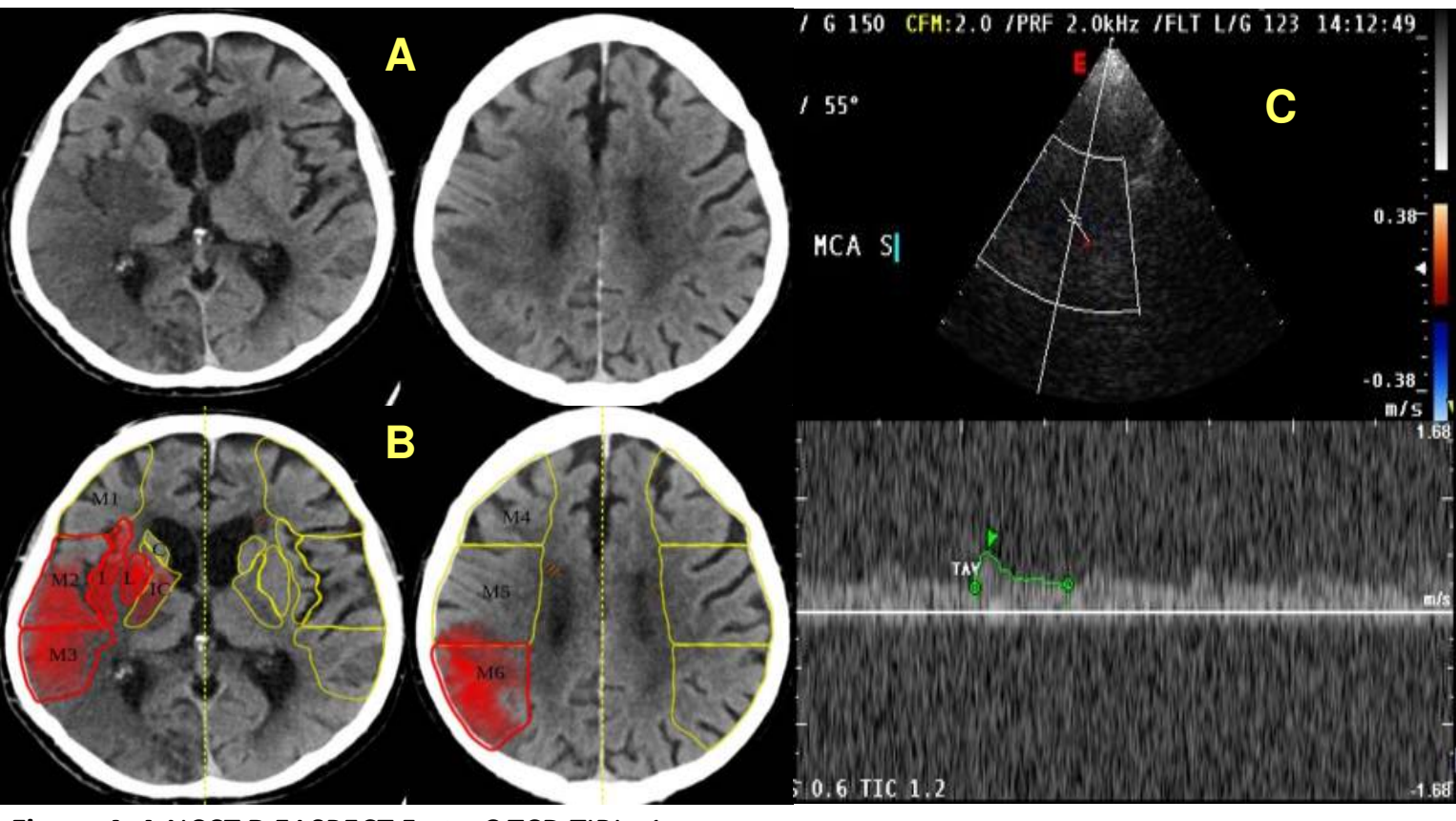

MCA flow was assessed with TCD/TCCD and scored with TIBI classification on admission and on the 7th day. ASPECT score was established for the CT performed on admission and after $24 \mathrm{~h}$ of follow up (with CT or DW-MRI) (Fig. $2,3,4)$.

Results: A significant correlation was found between ASPECT and the TIBI scores assessed on admission for both, all patients and only those with stroke $(r=0,23 ; p=0,04$ and $r=0,28 ; p=0,02$; respectively) (Fig. 5,6). In stroke patients, there were also associations between ASPECT score in the follow-up imaging and TIBI score assessed on admission and on the 7th day stroke $(r=0,25 ; p=0,04$ and $r=0,45 ; p<0,01$; respectively) (Fig. 7,8).
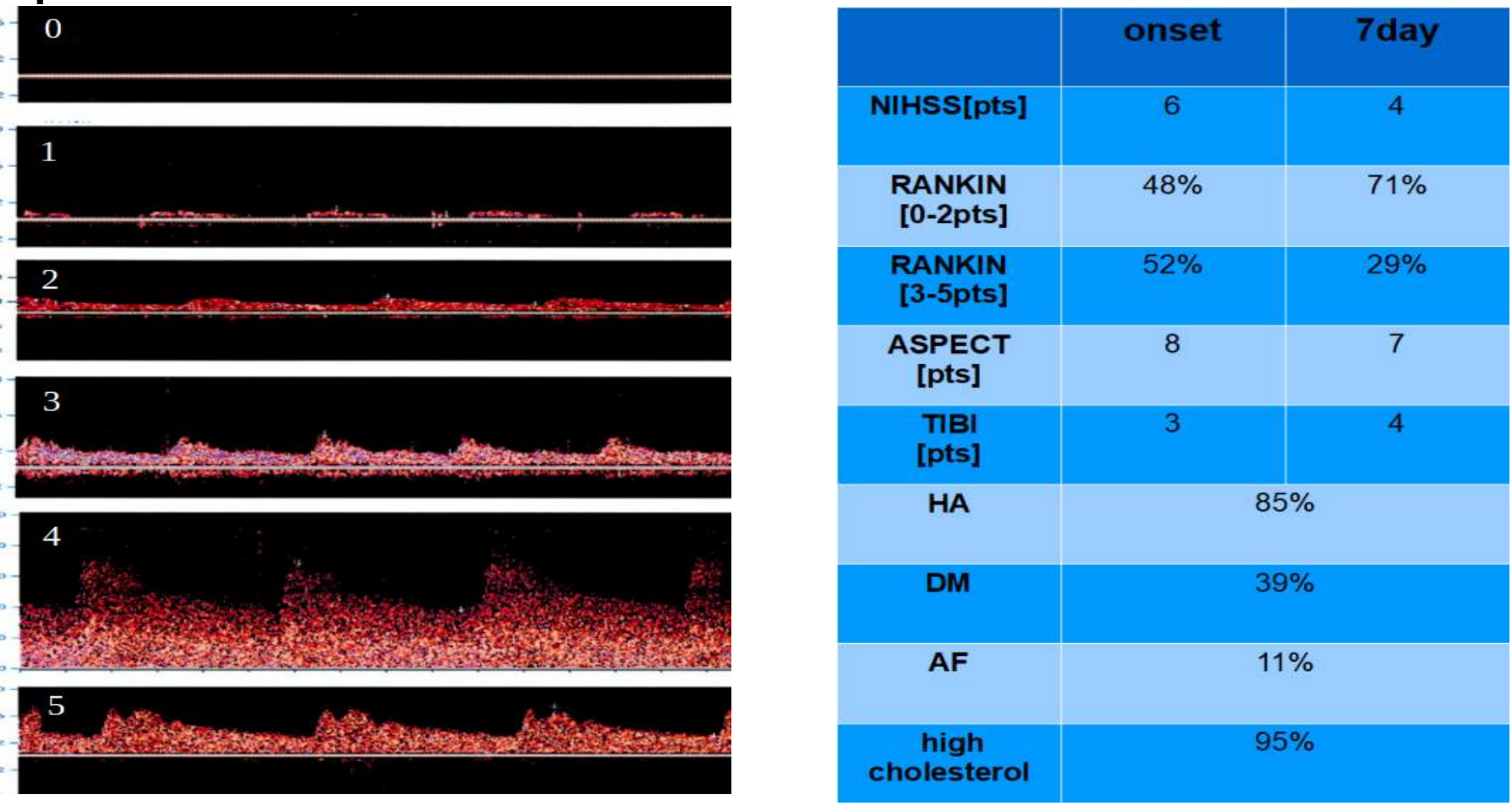

Figure 1. TIBI classification [by Alexandrov and al.]

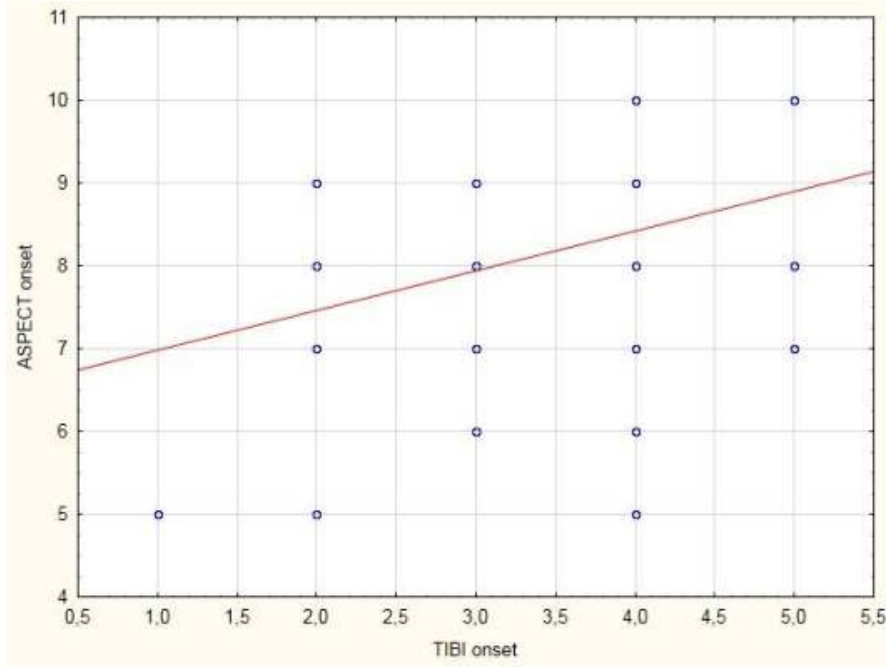

Figure 5. Correlation between ASPECT and the TIB scores on stroke onset (all patients).

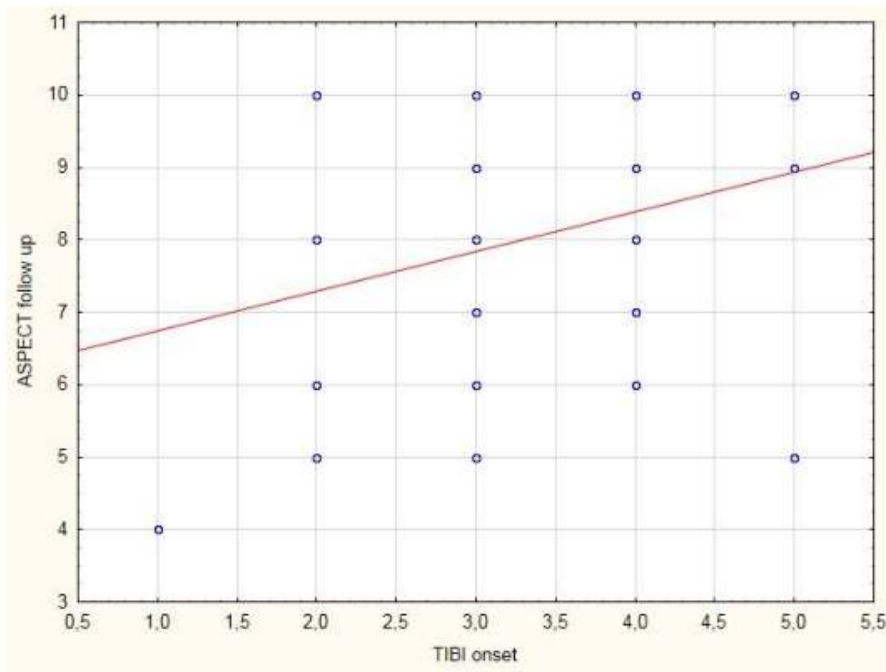

Figure 7. Correlation between follow up ASPECT and the TIBI scores on stroke onset.
Table 1. Neurological deficit, functional status and risk factors.

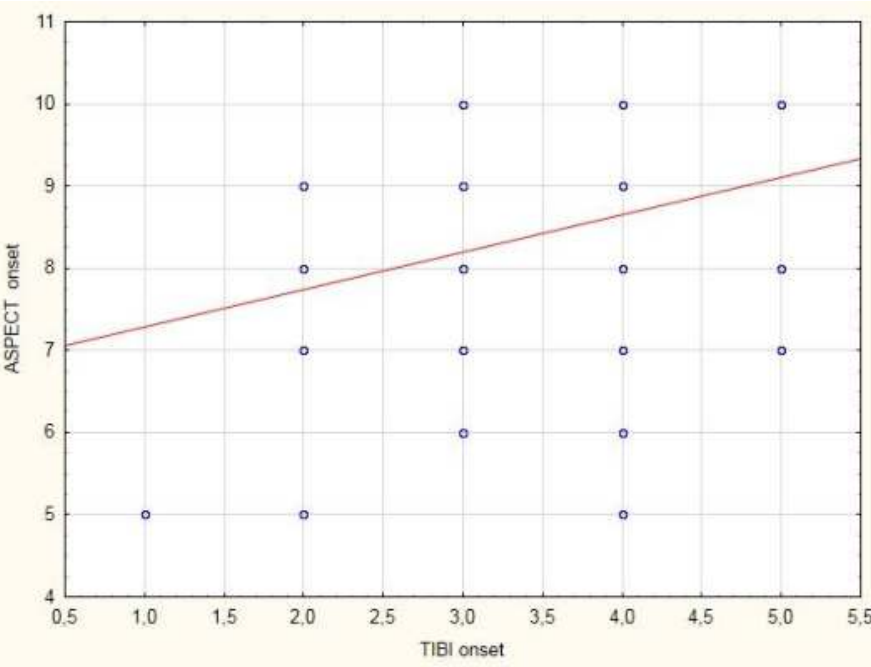

Figure 6. Correlation between ASPECT and the TIBI scores on stroke onse (stroke patients only).

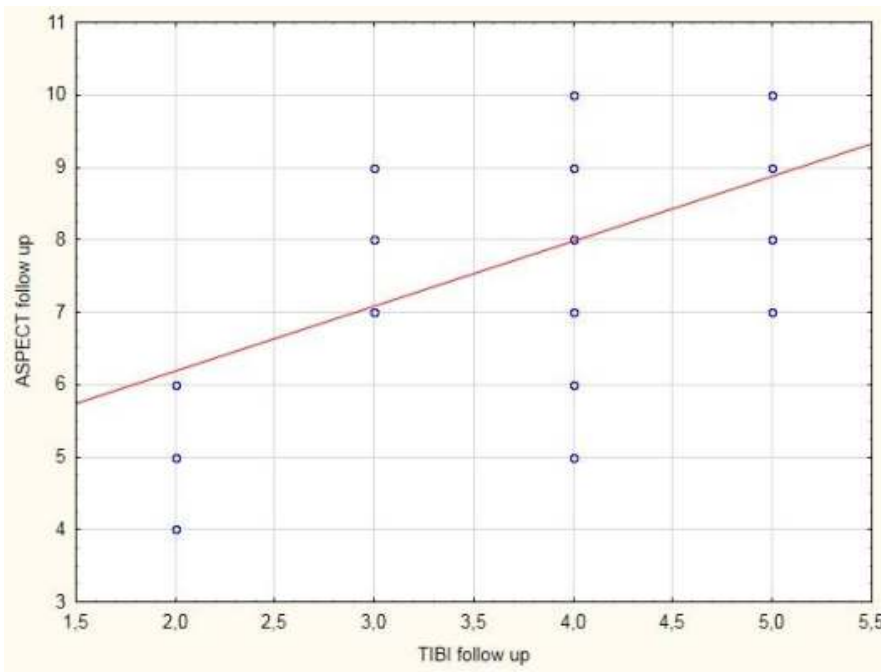

Figure 8. Correlation between follow up ASPECT and the TIBI scores on follow up. 